\title{
Linear flexural natural frequencies and stability analysis of spinning Rayleigh beams: application to clamped-clamped beams.
}

\author{
Mohamed Amine Aouadi, Faouzi Lakrad \\ Laboratory of Renewable Energy and Dynamics of Systems, Faculty of Sciences Ain Chock, \\ University Hassan II-Casablanca, Morocco
}

\begin{abstract}
In the present paper 3D bending linear free vibrations of spinning Rayleigh beams are investigated. Four linear models, that differ in the linearization process, are studied. A focus on analytical computation of natural frequencies for a broad range of boundary conditions is highlighted. Then, the conditions of occurrence of divergence and flutter instabilities are determined. Finally, a case study consisting of a clamped-clamped Rayleigh beam is studied. It is found that the free vibrations destabilization process depends on the used linearization approach.
\end{abstract}

\section{Introduction}

Spinning beams along their longitudinal axes are used in many space and mechanical structures and they are an important part of rotating machines [1] and [2]. Spinning can cause rigid body motions, axial, torsional and 3D flexural vibrations. Moreover, spinning introduces gyroscopic effects, including Coriolis, centrifugal, and Euler forces. This latter force appears when spinning velocity is nonconstant.

Aouadi and Lakrad [3] made a literature survey on 3D bending linear models of spinning Rayleigh beams. They focused on four models that differ according to the introduction stage of the linearization process in the computations. They expressed these four models in a condensed model and discussed the results of a double-hinged spinning Rayleigh beam.

The present paper is a continuation of the work [3]. Hence, the main contributions of the present work are :

1. Analytical derivation, in a general framework, of natural frequencies and stability analysis of the condensed model of 3D flexural vibrations of spinning Rayleigh beams [3].

2. Study of clamped-clamped spinning Rayleigh beams.

The present paper is organized as follows: in section 2, the mathematic model of spinning Rayleigh beams introduced in [3] is presented. In section 3, natural frequencies and stability analysis are investigated in general framework. In section 4, the clamped-clamped spinning Rayleigh beam is studied.

\section{Formulation of the problem}

The mathematical modelling used in the present paper is based mainly on the work [3].

The following assumptions are used

1. The Rayleigh beam theory is used.

2. The beam is straight in its undeformed state.
3. The beam has a uniform cross section, and uniform and isotropic material properties along its length.

4. Cross-sections of the beam have at least two axes of symmetry.

5. The effect of gravity and damping are not taken into account.

6. The spinning axe is the longitudinal direction of the undeformed beam.

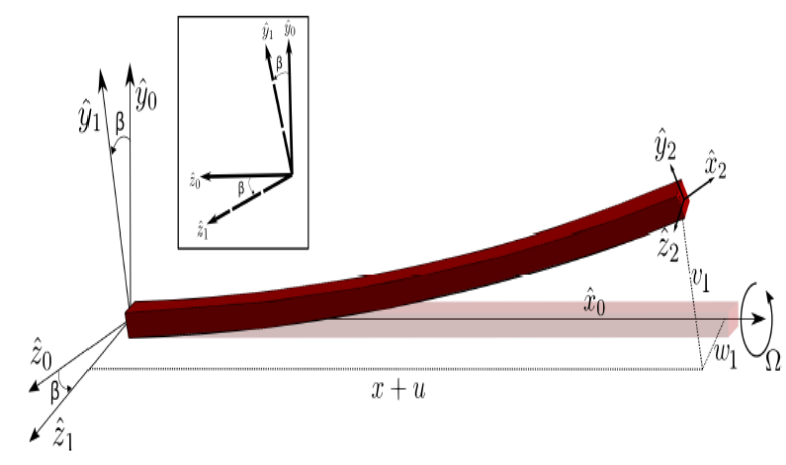

Fig. 1. A model of the spinning beam about its undeformed longitudinal direction.

Figure 1 shows the used mathematical model of the spinning beam. The axes $\left(x_{0}, y_{0}, z_{0}\right)$ and $\left(x_{2}, y_{2}, z_{2}\right)$ are assumed to be the principal axes of the initial undeformed and the final deformed configurations, respectively. The former axes are inertial and represent the fixed coordinate system describing the undeformed beam geometry. All the used frames have their origin on the centroid $C$ of the given cross section of the beam. Moreover, it is assumed that the static equilibrium of the beam corresponds to the situation where the longitudinal axes $x_{2}$ and $x_{0}$ are coincident [4]. Four frames $\mathcal{F}_{i}$, with $i=0, . ., 3$, are necessary to describe transformations from the initial state to the final deformed state [3].

The four approaches for linearizing the equations of motion reported in [3] are 
1. Approach 1: keeping all terms in the velocity vector that lead to quadratic terms in the kinetic energy.

2. Approach 2: keeping only linear terms in the velocity vector.

3. Approach 3: linearizing the Euler angles in the angular velocity vector.

4. Approach 4: linearizing the Euler angles in the velocity vector.

Approaches 1 and 4 can be seen from kinetic energy point of view as a late linearization methods and the others approaches as early linearization methods.

Equations of the transversal displacements $v_{1}$ and $w_{1}$ of the cross-section center along the rotated frame $\mathcal{F}_{1}$, corresponding to the four linearization approaches, can be written in the following compact non-dimensional form [3]

$$
\begin{gathered}
\ddot{v}_{1}-\gamma_{z} \ddot{v}_{1}^{\prime \prime}+v_{1}^{\prime \prime \prime \prime}- \\
-\overbrace{2 r \dot{w}_{1}}^{\text {Coriolisforce }}-\overbrace{\dot{r}\left(w_{1}+\left(1-\delta_{2 k}\right) \gamma_{y} w_{1}^{\prime \prime}\right)}^{\text {Eulerforces }} \\
\left.\ddot{w}_{1}-\gamma_{y} \ddot{w}_{1}^{\prime \prime}+\Gamma w_{1}^{\prime \prime \prime}+\left(\delta_{1 k} \gamma_{z}-2 \delta_{3 k} \gamma_{y}\right) v_{1}^{\prime \prime}\right) \\
+\overbrace{2 r \dot{v}_{1}}^{\text {Contrifugalforces }}+\overbrace{\dot{r\left(v_{1}-\left(1-\delta_{2 k}\right) \gamma_{y} v_{1}^{\prime \prime}\right)}}^{\text {Eulerforces }} \\
-\overbrace{r^{2}\left(w_{1}+\left(\delta_{1 k} \gamma_{y}+2 \delta_{3 k} \gamma_{z}\right) w_{1}^{\prime \prime}\right)}^{\text {Centrifugalforces }}=0(2)
\end{gathered}
$$

where $\delta_{i j}$ is the Kronecker delta, the dot and the prime are temporal and spatial derivatives, respectively. The other parameters of the model are

$-k$ is an integer that refers to the used linearization approach.

- $r$ is the ratio of the spinning velocity and a characteristic bending natural frequency of the beam.

$-\gamma_{y}$ and $\gamma_{z}$ are the inverse of the square of the slenderness about the respective axes.

$-\Gamma$ is the ratio of quadratic moments with respect to $y$ axis and $z$-axis. It measures the difference in the flexural rigidities in the two principal directions of the cross section. Moreover, $\Gamma=\gamma_{y} / \gamma_{z}$ since we are studying beams with cross-sections having at least two symmetric axes.

All the four approaches equations have in common the four first terms that correspond to the linear flexural vibrations of a non-spinning Rayleigh beam model and to the Coriolis force. The differences between all the approaches are in the Centrifugal and Euler forces. Moreover, all the four approaches lead to the same Euler-Bernoulli model when rotary inertia are cancelled i.e., $\gamma_{y}=\gamma_{z}=0$. Thus, the linearization process has no effect on the mathematical model in the case of Euler-Bernoulli beams [3].

Furthermore, the first and the third approaches have the same Euler forces terms that are caused by translational and rotational motions of the beam. The second approach has only the Euler force caused by the translational motion.

The four approaches have the same translational centrifugal forces and different contributions of the rotational motion of the beam. This latter does not contribute in the second approach when the velocity vector is taken linear.

The nonconstant spinning velocity causes the Euler forces

that act as parametric excitations of the rotating beam. In the case of constant spinning velocity, approaches 2 and 4 are giving the same equations of motion.

\section{Natural frequencies and stability analysis}

In this section natural frequencies of the spinning Rayleigh beams are computed in the case of constant spinning velocity i.e., $\dot{r}=0$. Thus, using separation of variables method, solutions of Eqs. (1) and (2) are sought in the following form

$$
\begin{aligned}
v_{1}(X, t) & =\sum_{n=1}^{N} q_{n}(t) \phi_{n}(X) \\
w_{1}(X, t) & =\sum_{n=1}^{N} p_{n}(t) \phi_{n}(X)
\end{aligned}
$$

where $\phi_{n}(X)$ is the nth bending normal mode shape of the beam for a given boundary conditions. Then, substituting Eqs. (3) and (4) into the partial differential equations (1) and (2) leads to the following system of ordinary differential equations that governs the temporal dependencies of the solutions

$$
\begin{aligned}
& \left(\phi_{n}-\gamma_{z} \phi_{n}^{\prime \prime}\right) \ddot{q}_{n}-2 r \phi_{n} \dot{p}_{n} \\
+ & {\left[\phi_{n}^{\prime \prime \prime \prime}-r^{2}\left(\phi_{n}+\left(\delta_{1 k} \gamma_{z}-2 \delta_{3 k} \gamma_{y}\right) \phi_{n}^{\prime \prime}\right)\right] q_{n}=0 } \\
& \left(\phi_{n}-\gamma_{y} \phi_{n}^{\prime \prime}\right) \ddot{p}_{n}+2 r \phi_{n} \dot{q}_{n} \\
+ & {\left[\Gamma \phi_{n}^{\prime \prime \prime \prime}-r^{2}\left(\phi_{n}+\left(\delta_{1 k} \gamma_{y}+2 \delta_{3 k} \gamma_{z}\right) \phi_{n}^{\prime \prime}\right)\right] p_{n}=0 }
\end{aligned}
$$

Applying the Galerkin method Eqs.(5) and (6) become

$$
\begin{aligned}
A_{n} \ddot{q}_{n}+B_{n} q_{n}-C_{n} \dot{p}_{n} & =0 \\
D_{n} \ddot{p}_{n}+E_{n} p_{n}+C_{n} \dot{q}_{n} & =0
\end{aligned}
$$

Combining Eqs.(7) and (8) we obtain the following fourth order ordinary differential equation

$$
\ddot{\ddot{\alpha}}_{n}+\eta_{n} \ddot{\alpha}_{n}+\zeta_{n} \alpha_{n}=0
$$

where the variable $\alpha_{n}=q_{n}(t)$ or $p_{n}(t)$ and the parameters of Eq. (9) are given by

$$
\eta_{n}=\frac{B_{n} D_{n}+C_{n}^{2}+A_{n} E_{n}}{A_{n} D_{n}} \quad ; \quad \zeta_{n}=\frac{B_{n} E_{n}}{A_{n} D_{n}}
$$

where

$$
\begin{aligned}
A_{n} & =\int_{0}^{1}\left(\phi_{n}-\gamma_{z} \phi_{n}^{\prime \prime}\right) \phi_{n} d x \\
B_{n} & =\int_{0}^{1}\left[\phi_{n}^{\prime \prime \prime \prime}-r^{2}\left(\phi_{n}+\left(\delta_{1 k} \gamma_{z}-2 \delta_{3 k} \gamma_{y}\right) \phi_{n}^{\prime \prime}\right)\right] \phi_{n} d x \\
C_{n} & =\int_{0}^{1} 2 r \phi_{n}^{2} d x=2 r \\
D_{n} & =\int_{0}^{1}\left(\phi_{n}-\gamma_{y} \phi_{n}^{\prime \prime}\right) \phi_{n} d x \\
E_{n} & =\int_{0}^{1}\left[\Gamma \phi_{n}^{\prime \prime \prime \prime}-r^{2}\left(\phi_{n}+\left(\delta_{1 k} \gamma_{y}+2 \delta_{3 k} \gamma_{z}\right) \phi_{n}^{\prime \prime}\right)\right] \phi_{n} d x
\end{aligned}
$$


The time dependent solutions are sought in the following form

$$
\begin{aligned}
q_{n}(t) & =a_{1 n} \cos \left(\omega_{n}^{+} t\right)+a_{2 n} \sin \left(\omega_{n}^{+} t\right)+a_{3 n} \cos \left(\omega_{n}^{-} t\right) \\
& +a_{4 n} \sin \left(\omega_{n}^{-} t\right) \\
p_{n}(t) & =b_{1 n} \cos \left(\omega_{n}^{+} t\right)+b_{2 n} \sin \left(\omega_{n}^{+} t\right)+b_{3 n} \cos \left(\omega_{n}^{-} t\right) \\
& +b_{4 n} \sin \left(\omega_{n}^{-} t\right)
\end{aligned}
$$

where the unknown amplitudes $a_{\text {in }}$ and $b_{\text {in }}$ (with $i=1, . ., 4$ ) are obtained through initial conditions.

Finally, the natural frequencies $\omega_{n}^{ \pm}$of Rayleigh spinning beams, in general framework, are given by

$$
\omega_{n}^{ \pm}=\frac{1}{\sqrt{2}} \sqrt{\eta_{n} \pm \sqrt{\eta_{n}^{2}-4 \zeta_{n}}}
$$

The natural frequencies depend strongly on the boundary conditions through the bending mode shape $\phi_{n}(X)$. Furthermore, non-spinning bending frequencies of Rayleigh beams can be obtained from Eq.(17) by cancelling the spinning angular velocity i.e., $r=0$.

Moreover, bending natural frequencies of spinning EulerBernoulli beams can be obtained from Eq.(17) by setting $\gamma_{y}=\gamma_{z}=0$.

The temporal dependence $\alpha_{n}(t)$ of the nth mode is independent of the other modes. Thus, to perform a stability analysis, the fourth order equation (9) can be rewritten on the form of a system of four first order ODEs. The eigenvalues $\lambda$ of the corresponding Jacobian matrix are given by the following characteristic equation

$$
\lambda^{4}+\eta_{n} \lambda^{2}+\zeta_{n}=0
$$

The stability of the nth mode of the spinning beam is determined by examining the signs of real parts of the four $\lambda$ solutions of Eq. (18). Hence, the divergence instability occurs for $\zeta_{n}=0$ and the flutter instability occurs for $\eta_{n}^{2}-4 \zeta_{n}=0$.

\section{Case study : clamped-clamped spinning beam}

Approaches 1 and 2 will be applied to the spinning clampedclamped Rayleigh beam. This case was studied by Sheu and Yang [5] and Pai et al. [6] using the approach 2 and the equations of motion were expressed in the inertial frame. The case of spinning Euler-Bernoulli beams was studied earlier by Bauer [7].

The boundary conditions corresponding to the clampedclamped case are

$$
\begin{aligned}
& v_{1}(0, t)=w_{1}(0, t)=v_{1}^{\prime}(0, t)=w_{1}^{\prime}(0, t)=0 \\
& v_{1}(1, t)=w_{1}(1, t)=v_{1}^{\prime}(1, t)=w_{1}^{\prime}(1, t)=0
\end{aligned}
$$

The natural bending modes shapes of the clamped-clamped Euler-Bernoulli non spinning beam are used as the spatial dependence of the solutions. Thus,

$$
\begin{aligned}
\phi_{n}(X) & =\left[\cos \left(\beta_{n} X\right)-\cosh \left(\beta_{n} X\right)\right. \\
& \left.-\frac{\left(\cos \left(\beta_{n}\right)-\cosh \left(\beta_{n}\right)\right)\left(\sin \left(\beta_{n} X\right)-\sin \left(\beta_{n} X\right)\right)}{\sin \left(\beta_{n}\right)-\sinh \left(\beta_{n}\right)}\right]
\end{aligned}
$$

where $\beta_{n}$ is the nth positive solution of the following algebraic equation : $\cos (\beta) \cosh (\beta)=1$.

The first four modes shapes of the $3 \mathrm{D}$ bending vibration of the spinning beam corresponding to Eq.(21) are shown in Fig. 2.

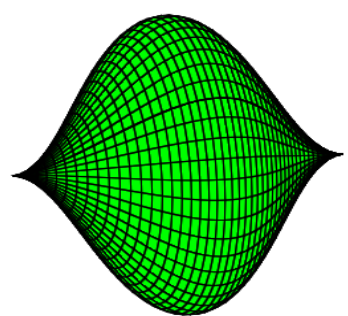

(a) Mode 1 shape

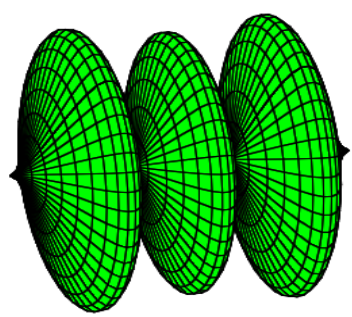

(c) Mode 3 shape

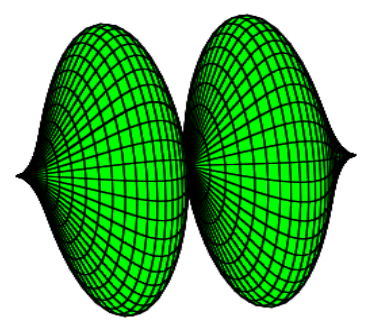

(b) Mode 2 shape

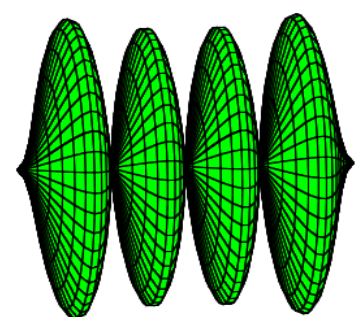

(d) Mode 4 shape
Fig. 2. First four modes shapes of 3D bending vibration of a spinning beam.

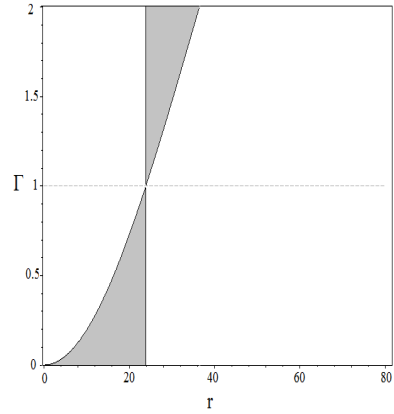

(a) Approach $1: \gamma_{z}=0.01$

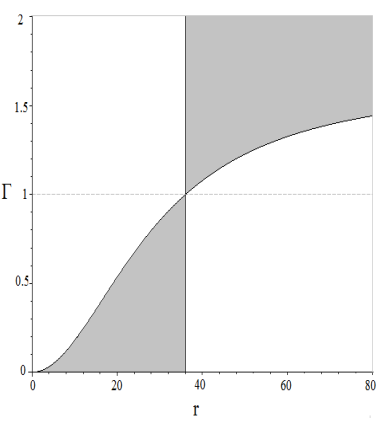

(c) Approach $1: \gamma_{z}=0.05$

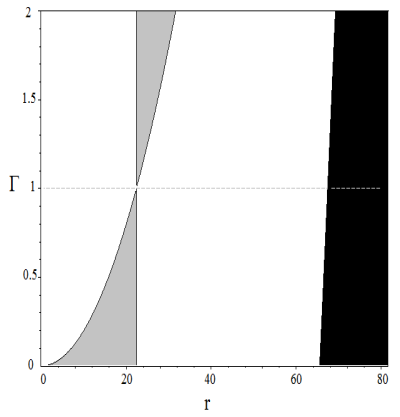

(b) Approach $2: \gamma_{z}=0.01$

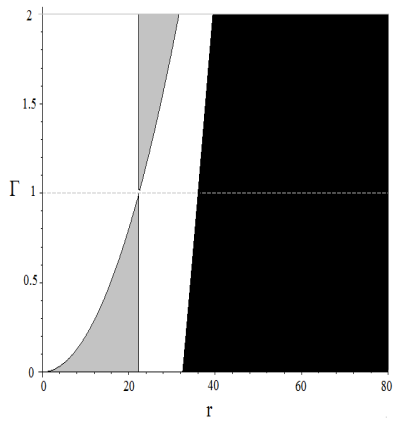

(d) Approach 2: $\gamma_{z}=0.05$
Fig. 3. Stability charts of the clamped-clamped spinning Rayleigh beam for $\mathrm{n}=1$ in the $(r, \Gamma)$-plane. The grey zone: divergence instability, the black zone: flutter instability. The left curves correspond to the approach 1 and right curves to the approach 2 . 
In Fig. 3 are shown the stability charts of clampedclamped spinning Rayleigh beams, for the first mode $\mathrm{n}=$ 1 , given by approaches 1 and 2, respectively. All these figures show that increasing $\gamma_{z}$ i.e., decreasing the slenderness ratio parameter leads to the increase of the zones of instability (grey and black zones). Moreover, the divergence instability zone is composed of two parts that shrinks to a point at $\Gamma=1$. All eigenvalues of this shrinking point are equal to zero.

Moreover, the approach 1 results exhibit only divergence instability, see Figs. 3a and 3c. Approach 2 exhibits both divergence and flutter instabilities, see Figs. $3 \mathrm{~b}$ and $3 \mathrm{~d}$. However, its zone of divergence instability is independent of $\gamma_{z}$ and is similar to the instability zone of the EulerBernoulli beam. The zone of flutter instability is increasing with increasing $\gamma_{z}$. The same results were found in the case of double-hinged Rayleigh beam [3].

In Fig. 4, the non-dimensional bending first natural frequencies of spinning Rayleigh and Euler-Bernoulli beams are plotted versus the non-dimensional spinning velocity $r$, using the linearization approaches 1 and 2 , for $\gamma_{z}=0.01$ and $\Gamma=0.5$. Initially, the non spinning beams have two bending fundamental frequencies caused by the difference in bending rigidities in the $y$ and $z$ axes. Furthermore, $\omega_{1}^{+}$ is the upper curve that increases with increasing $r$. The frequency $\omega_{1}^{-}$is decreasing till it destabilizes by a divergence instability. Then, it stabilizes again for $r \approx 22$ and increases with increasing $r$. This scenario will continue for higher values of $r$ in the case of approach 1. For the approach 2, the two fundamental frequencies of the spinning Rayleigh beam destabilize through a flutter instability. Frequencies of spinning Euler-Bernoulli beams do not exhibit a flutter instability.

\section{Conclusion}

In the present work the compact model developed in [3] is investigated. This model includes four linearization approaches of 3D bending linear vibrations of spinning Rayleigh beams. The natural frequencies and the instability scenarios of the free vibrations are determined in a general framework for a broad range of boundary conditions. The clampedclamped spinning Rayleigh beam was taken as a case study. It is found that two linearization approaches are leading to different processes of destabilization of the free vibrations. The approach 1 exhibits only the divergence instability and the approach 2 exhibits divergence and flutter instabilities. Only the experimental data will be able to assess the degree of correctness of the different theoretical models.

\section{References}

1. J.S. Rao. History of rotating machinery dynamics. Springer, 2011.

2. D.F. de Castro, I.A.A. Prado, M.F.V. Pereira, D.A. Santos, J.M. Balthazar. In-Flight Parameter Estimation for Multirotor Aerial Vehicles. MATEC Web of Conferences 83, 01004, 2016

3. M.A. Aouadi, F. Lakrad. On mathematical modelling of linear flexural vibrations of spinning Rayleigh beams. J. Sound Vib. 430, 17-35, 2018.

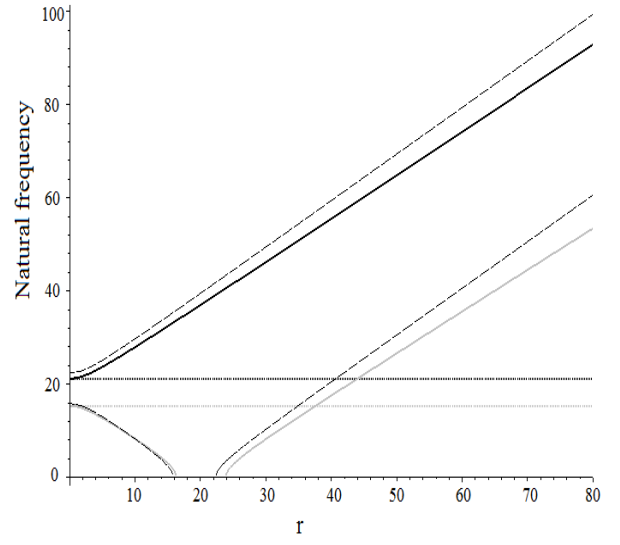

(a) Approach 1

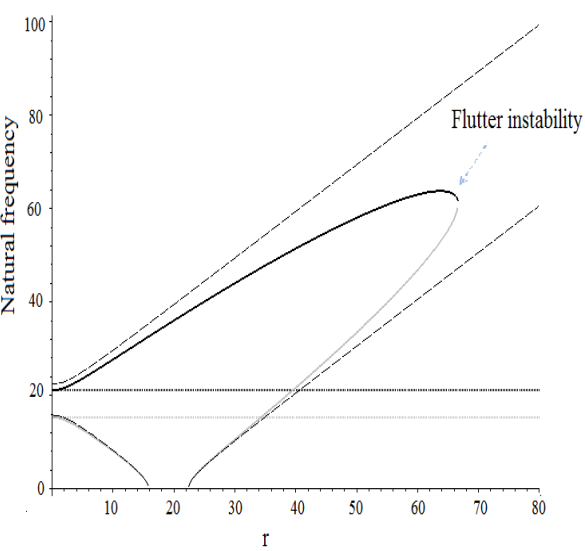

(b) Approach 2

Fig. 4. First natural frequency of clamped-clamped beam versus the spinning speed $r$, for $\gamma_{z}=0.01$ and $\Gamma=0.5$. The continuous lines: Rayleigh spinning beam. Dashed lines : Euler-Bernoulli spinning beam. Dotted lines: non-spinning Rayleigh beam.

4. A.H. Nayfeh, P.F. Pai. Linear and Nonlinear StructuralMechanics, Wiley-Interscience, New York, 2004.

5. G.J. Sheu, S.M. Yang. Dynamic analysis of a spinning Rayleigh beam, Int. J. Mech. Sci. 47, 157-169, 2005.

6. P.F. Pai, X. Qian, X. Du. Modeling and dynamic characteristics of spinning Rayleigh beams. Int. J. Mch. Sci. 68, 291-303, 2013.

7. H.F. Bauer. Vibration of rotating uniform beam. Part I: orientation in the axis of rotation, J. Sound Vib. 72, 177-189, 1980. 